\title{
ON SMOOTHING OPERATIONS AND THEIR GENERATING FUNCTIONS ${ }^{1}$
}

\author{
I. J. SCHOENBERG
}

\section{INTRODUCTION}

In this paper we are mainly concerned with two kinds of linear transformations, the sequence convolution transformation

$$
y_{n}=\sum_{\nu=-\infty}^{\infty} a_{n-\nu} x_{\nu}
$$

and the integral convolution transformation

$$
g(x)=\int_{-\infty}^{\infty} \Lambda(x-t) f(t) d t
$$

where the sequence $\left\{a_{n}\right\}$ and the function $\Lambda(x)$ are thought of as given. In $\$ 2$ we also consider the ordinary linear transformation

$$
y_{i}=\sum_{k=1}^{n} a_{i k} x_{k} \quad(i=1, \cdots, m) .
$$

The loosely connected topics to be discussed concerning these transformations are perhaps best brought together under the general subject of smoothing operations.

In $\S 1$ we are concerned with transformations (1) of the kind used for the purpose of smoothing numerical data. Erastus L. De Forest proposed long ago the problem of describing the asymptotic behavior of the coefficients of high-order iterates of (1) (see Wolfenden [15]). This question, as well as the question of when a formula (1) may rightly be called a smoothing formula, was answered by the author in [38] and [31]. It is shown in $\$ 1$ that the author's criterion for a smoothing formula is essentially of the nature of a stability condition of the kind required of difference methods for the numerical integration of partial differential equations. From this point of view De Forest's problem amounts to constructing by difference methods the fundamental solutions of certain parabolic differential equations. These remarks are merely special cases of Fritz John's recent work

An address delivered before the Fresno meeting of the Society on May 3, 1952 by invitation of the Committee to Select Hour Speakers for Far Western Sectional Meetings; received by the editors June 30, 1952.

1 This work was performed on a National Bureau of Standards contract with the University of California, Los Angeles and was sponsored (in part) by the Office of Scientific Research, USAF. 
[49] on the integration of parabolic differential equations.

The remainder of the paper discusses those transformations (1) and (2) which are variation-diminishing. These special classes of smoothing operations, while perhaps too narrow for practical smoothing purposes (because, in actuarial terms, they preserve only linear functions), seem to deserve particular attention because of the interesting problems which arise if we attempt to characterize their respective generating functions

$$
\sum_{-\infty}^{\infty} a_{n} z^{n} \text { and } \int_{-\infty}^{\infty} e^{-x s} \Lambda(x) d x .
$$

$\S \S 3$ and 4 are devoted to these problems concerning (2) and (1), respectively. A discussion of (2), initiated by Pólya in 1915, has recently received considerable attention by Widder, Hirschman, and the author. The transformation (1), studied by M. Aissen, Anne Whitney and the author, is the subject of very significant as yet unpublished contributions at the hands of Albert Edrei (\$4). Edrei's results are used to establish (Article 4.3) a conjecture of Schoenberg [31, Part B, pp. 131-132].

Our discussion in $\$ 2$ of the transformation (3) is mainly intended to provide a background for the transcendental cases of (1) and (2). Attention is called to a recent theorem of R. Gantmakher and M. Krein which is of interest in the present connection and is also helpful in a discussion of curves which are convex in higher-dimensional spaces. $^{2}$

\section{The stability of SMOOTHING FORMULAE}

\subsection{What is a smoothing formula? Let}

$$
y_{m}=\sum_{\nu=-\infty}^{\infty} a_{m-\nu} x_{\nu}
$$

be a given "moving average" formula which is such that the function

$$
F(z)=\sum_{-\infty}^{\infty} a_{\nu} z^{\nu} \quad\left(r<|z|<r^{-1}\right),
$$

which may be called its generating function, is regular in an open ring

${ }^{2}$ This remark explains the presence of several geometrical papers in our bibliography. As seen from this brief description of contents, the paper is concerned with the most general smoothing operations on the one hand $(\$ 1)$, with a very restricted class on the other $(\$ \S 3,4)$. For the middle-range of operations which are useful in the practice of smoothing data we refer to the excellent expositions by WhittakerRobinson [17], and Wolfenden [15]. For the related subject of osculatory interpolation see Greville [29] and Schoenberg [31]. 
containing the circle $|z|=1$. Let us assume, as is generally the case in practice, that the given sequence of "weights" $a_{m}$ is symmetric about $a_{0}$ :

$$
a_{m}=a_{-m} \quad(m=1,2, \cdots) .
$$

It was pointed out in [31] that the generating function (1.2) or, equivalently, the characteristic function

$$
\phi(u)=F\left(e^{i u}\right)=a_{0}+2 a_{1} \cos u+2 a_{2} \cos 2 u+\cdots
$$

may be used to advantage in describing some of the useful attributes of a moving average formula (1.1). Thus the formula (1.1) will leave unchanged, or invariant, sequences $\left\{x_{m}\right\}$ which are equidistant ordinates of polynomials of degree $2 k-1$, but of no higher degree, provided $\phi(u)$ has an expansion in powers of $u$ which is of the form

$$
\phi(u)=1-\lambda u^{2 k}+\cdots \quad(\lambda \neq 0) .
$$

Good arguments were presented in [31] and still better ones in [38] to the effect that a moving average formula (1.1) could be called a smoothing formula only if (perhaps also if) its characteristic function satisfies the condition

$$
|\phi(u)|<1 \quad \text { if } 0<u<2 \pi .
$$

This condition implies that the coefficient $\lambda$ of (1.5) is positive. ${ }^{3}$

1.2. Behavior of the iterates of (1.1): De Forest's problem. A conclusive argument in support of the necessity of our condition (1.6) is furnished by the solution of the following problem first stated and attacked by Erastus L. De Forest. ${ }^{4}$ If we subject the given se-

${ }^{3}$ During his connection in the last war with the Data Analysis Section of the Boeing Aircraft Company, Dr. C. Lanczos has reached the same criterion (1.6) from the following point of view: If the formula (1.1) is applied to the simple vibration of frequency $u, x_{\nu}=e^{i u \nu}(-\infty<\nu<\infty)$, we obtain the transformed sequence $y_{m}=e^{i u m} \phi(-u)(-\infty<m<\infty)$, which is again a simple vibration of frequency $u$, of amplitude $|\phi(-u)|$. Requiring that the amplitude of every simple vibration with frequency in the range $0<u<2 \pi$ be diminished in the process, Dr. Lanczos reached our condition (1.6). This interesting remark shows that the values of $\phi(u)$ play the role of characteristic values of the matrix of the linear operation (1.1). The condition (1.6) is now seen to be related to the conditions found by R. Oldenburger and A. Dresden (for references see [47]) which insure that the $n$th power of an ordinary matrix converges to a limit as $n$ tends to infinity.

${ }^{4}$ Erastus L. De Forest (1834-1888) contributed numerous original and fundamental papers on various aspects of the problem of fitting and smoothing irregular series by means of polynomials. His pioneer work remained practically unknown, however, until 1924, when its importance in relation to the graduation processes of actuaries was described by Hugh $\mathrm{H}$. Wolfenden in his paper [15]. A further summary of De Forest's contributions can be found in H. H. Wolfenden, The fundamental principles of mathematical statistics, Toronto, Macmillan, 1942. 
quence $\left\{x_{m}\right\} n$ times in succession to the same transformation (1.1), we obtain a linear transformation

$$
y_{m}^{(n)}=\sum_{\nu=-\infty}^{\infty} a_{m-\nu}^{(n)} x_{\nu}
$$

which is the $n$-fold iterate of (1.1). What is the asymptotic behavior of the coefficients of (1.7) as $n \rightarrow \infty$ ? This question was answered for the case when all coefficients of (1.1) are non-negative, hence necessarily that $k=1$ in (1.5), by De Forest and by G. B. Dantzig [26]. ${ }^{.} \mathrm{A}$ general solution is as follows:

Let (1.1) be such that (1.3), (1.6), and (1.5) are satisfied, hence $\lambda>0$. Let

$$
G_{k}(x)=\frac{1}{2 \pi} \int_{-\infty}^{\infty} e^{-v^{2 k}} \cos v x d v,
$$

which is the normal function

$$
G_{1}(x)=\frac{1}{2 \pi^{1 / 2}} e^{-x^{2} / 4}
$$

if $k=1$, otherwise $(k=2,3, \cdots)$ an entire even function having infinitely many zeros, all real. The coefficients of (1.7) satisfy the asymptotic relations

$$
a_{\nu}^{(n)}=(\lambda n)^{-1 / 2 k} G_{k}\left(\nu(\lambda n)^{-1 / 2 k}\right)+o\left(n^{-1 / 2 k}\right), \quad \text { as } n \rightarrow \infty,
$$

where the "little o" symbol holds uniformly for all integers $\nu$ (see Schoenberg [38]). It was also shown there by examples that (1.10) no longer holds if the equality sign is allowed in (1.6), and that the coefficient $a_{0}^{(n)}$ diverges exponentially to $+\infty$, as $n=2 m$ tends to infinity through even values, if the inequality (1.6) is reversed anywhere in the range $(0,2 \pi)$.

\footnotetext{
${ }^{5}$ The following additional references, which I owe to T. S. Motzkin, came too late to my attention for inclusion in our bibliography: The case of non-negative weights $(k=1)$ in the more general form where the convoluted formulae need not be identical was treated by Eugen Slutzky in a Russian paper of 1927. See its English translation The summation of random causes as the source of cyclic processes, Econometrica vol. 5 (1937) pp. 105-146, in particular pages 134-138. Concerning the same problem see also R. v. Mises, Wahrscheinlichkeitsrechnung und ihre Anwendungen in der Statistik und theoretischen Physik, Leipzig and Wien, 1931, and R. v. Mises, Generalizzazione di un teorema sulla probabilita della somma di un numero illimitato di variabili casuali, Giornale dell' Istituto Italiano degli Attuari vol. 5 (1934) pp. 483-
} 495. 
A corresponding discussion of smoothing of periodic data is related to the so-called finite Fourier series (see Schoenberg [47]). Some unpublished work by Fritz John deals with the analogue of (1.10) for the periodic case.

1.3. A differential equation. The connection with differential equations is briefly as follows. Observe that (1.8) implies

$$
U(x, t)=t^{-1 / 2 k} G_{k}\left(x t^{-1 / 2 k}\right)=\frac{1}{2 \pi} \int_{-\infty}^{\infty} e^{-t v^{2 k}+i x v} d v \quad(t>0) .
$$

The function under the integral sign is immediately seen to satisfy, for all $v$, the differential equation

$$
\frac{\partial u}{\partial t}=(-1)^{k+1} \frac{\partial^{2 k} u}{\partial x^{2 k}},
$$

which reduces to the familiar heat-equation if $k=1$. It follows that also $U(x, t)$, defined by (1.11), is a solution of (1.12) in the upper half-plane $t>0$. On the other hand, applying to (1.8) Fourier's inversion formula and setting $v=0$, we find that

$$
\int_{-\infty}^{\infty} G_{k}(x) d x=1
$$

These remarks imply the following: If $f(x)$ is continuous and $o\left(|x|^{-2}\right)$, say, as $|x| \rightarrow \infty$, then

$$
u(x, t)=t^{-1 / 2 k} \int_{-\infty}^{\infty} G_{k}\left((x-v) t^{-1 / 2 k}\right) f(v) d v
$$

is a solution of the differential equation (1.12) satisfying the boundary condition

$$
\lim _{t \rightarrow+0} u(x, t)=f(x) .
$$

This particular solution $u(x, t)$ may now also be constructed as follows: Draw in the $(x, t)$-plane the lattice of points

$$
(\nu \Delta x, n \Delta t) \quad(\nu=0, \pm 1, \cdots ; n=0,1, \cdots) .
$$

Define in it a lattice-function $u_{\nu, n}$ by starting with

$$
u_{\nu, 0}=f(\nu \Delta x)
$$

and computing the values along each horizontal line from those on the line below by means of (1.1). This evidently amounts to iterating (1.1), so that we have 


$$
u_{\nu, n}=\sum_{\mu=-\infty}^{\infty} a_{\nu-\mu}^{(n)} f(\mu \Delta x)
$$

For any given $x$ and $t>0,(1.14)$ goes over into (1.13) under the following circumstances: We first connect the mesh-sizes $\Delta x$ and $\Delta t$ by the relation

$$
\Delta t=\lambda(\Delta x)^{2 k} .
$$

If the integers $\nu$ and $n$ are such that

$$
\nu \Delta x \rightarrow x \text { and } n \Delta t \rightarrow t, \text { as } \Delta x \rightarrow+0,
$$

then

$$
u_{\nu, n} \rightarrow u(x, t) .
$$

This follows readily from (1.14) and (1.13), in view of the asymptotic relations (1.10): (1.14) differs from a Cauchy-Riemann sum for the integral (1.13) by a quantity which tends to zero because of the uniformity in $\nu$ of the error term of (1.10).

It is interesting to note that it matters little which formula (1.1) we use in this construction, as long as it preserves polynomials of degree $2 k-1$ and of no higher degree, i.e. satisfies (1.5), and above all that it satisfy the stability condition (1.6), the term "stability" meaning here stability on iteration. For the general theory of Fritz John of which the above situation (for $k=1$ ) is merely a very special example, see [49].

\section{VARIATION-DIMINISHING LINEAR TRANSFORMATIONS}

2.1. Fundamental criteria. Let

$$
y_{i}=\sum_{k=1}^{n} a_{i k} x_{k} \quad(i=1, \cdots, m)
$$

be a linear transformation where coefficients and variables are all real. Let $v(x)$ denote the number of changes of sign in the sequence $x_{k}$ and $v(y)$ the similar number for $y_{i}$. F. Klein discussed the problem of comparing the upper bounds for the number of real roots of an algebraic equation in a given interval, as furnished by the Descartes rule of signs and similar theorems. These bounds are usually equal to the number of changes of sign of sequences arising from the coefficients of the given equation by linear transformations. For this reason, Klein's problem led Schoenberg $[19 ; 21]$ to study transformations (2.1) which have the property that

$$
v(y) \leqq v(x),
$$


for all values of the variables $x_{k}$. Such transformations were called variation-diminishing by Pólya.

The matrix $A=\left\|a_{i k}\right\|$ is called totally positive, provided all its minors of all orders are non-negative [19, p. 322]. A useful criterion for such matrices, based on a lemma of M. Fekete [4, pp. 4-6], was recently given by Anne Whitney [58]. Totally positive squareshaped matrices were thoroughly investigated by Gantmakher-Krein $[24 ; 44]$ in connection with boundary-value problems arising in vibration problems. Schoenberg [19] showed that (1.1) is variation-diminishing if $A$ is totally positive. Assuming that the matrix $A$ is of rank $n$, he also proved the following: (1.1) is variation-diminishing if and only if $A$ enjoys the property that two minors of $A$, of equal orders, are never of opposite signs. If the rank $r$ of $A$ is unrestricted, Motzkin [22] proved that (1.1) is variation-diminishing if and only if two minors of $A$, of the same order $s$, are never of opposite sign if $s<r$, while if $s=r$ they should never be of opposite signs if they belong to the same set of $r$ columns of $A$. For a proof of this theorem, differing from Motzkin's, see Schoenberg-Whitney [51]. There also cyclic variation-diminishing transformations are characterized: (1.1) is said to be cyclic variation-diminishing provided it is such that the inequality

$$
v_{c}(y) \leqq v_{c}(x)
$$

always holds, where $v_{c}(x)$ is the number of changes of sign in the sequence $x_{1}, \cdots, x_{n}$ counted after these variables are placed cyclically along a circle, with a similar definition for $v_{c}(y)$.

2.2. A theorem of Gantmakher-Krein and convex curves. Let us assume that the rank of the matrix $A$, of (2.1), is equal to $n$. Choose $n$ among the linear forms $y_{i}$ which are linearly independent. As their values may be chosen at wish, for instance alternating in sign, it is clear that $\sup _{x} v(y) \geqq n-1$. The theorem of Gantmakher-Krein answers the question as to when we have the equality sign in this relation and may be stated as follows:

The system (1.1), of rank equal to $n$, is such that we always have

$$
v(y) \leqq n-1
$$

if and only if all nonvanishing minors of order $n$, of $A$, are of the same sign. See Gantmakher-Krein [44, Theorem 3 on p. 297], and also Schoenberg-Whitney [51, Theorem 1 on p. 142]. ${ }^{6}$

${ }^{6}$ Anne Whitney and the author were unaware of the book by Gantmakher and Krein when [51] was published. However, the priority clearly belongs to the Russian authors. 
This theorem seems fundamental for two reasons: Firstly, it implies easily the results of Schoenberg and Motzkin concerning variation-diminishing transformations and plays a similar role in a discussion of the related problem (2.3). Secondly, it seems indispensable in a discussion of curves which are convex in higher-dimensional spaces. The use of the term "convex" in this connection requires some explanation. We mention first the concept of an arc in $E_{m}$ of (linear) order $m$, due to C. Juel, intensively studied and generalized by O. Haupt, A. Marchaud [18], P. Scherk [25], and others: A Jordan arc in $E_{m}$ is of order $m$ provided it is intersected by every hyperplane in at most $m$ points. A related but distinct notion is as follows: A continuous arc

$$
\Gamma: x_{i}=x_{i}(t) \quad(i=1, \cdots, m ; \alpha \leqq t \leqq \beta)
$$

in the euclidean space $E_{m}$ is said to be convex in $E_{m}$ if it crosses every hyperplane of $E_{m}$ at most $m$ times. If we also require that $\Gamma$ should not be contained in a lower-dimensional flat space, then we say that $\Gamma$ is convex on $E_{m}$ (see Schoenberg [56].)

The following criterion is an almost immediate corollary of the theorem of Gantmakher-Krein: The arc $\Gamma$, defined by (2.5), is convex on $E_{m}$ if and only if the determinants

$$
\left|\begin{array}{cccc}
1 & x_{1}\left(t_{0}\right) & \cdots & x_{m}\left(t_{0}\right) \\
1 & x_{1}\left(t_{1}\right) & \cdots & x_{m}\left(t_{1}\right) \\
\vdots & \vdots & \vdots \\
1 & x_{1}\left(t_{m}\right) & \cdots & x_{m}\left(t_{m}\right)
\end{array}\right| \quad\left(\alpha \leqq t_{0}<t_{1}<\cdots<t_{m} \leqq \beta\right)
$$

do not all vanish and the nonvanishing among them are all of the same sign. The case when $m=2$ seems intuitively obvious, for the theorem says that the plane arc $P=P(t)$ is convex on $E_{2}$ provided the nondegenerate ones among the triangles $P\left(t_{0}\right) P\left(t_{1}\right) P\left(t_{2}\right)$ have all the same orientation. All convex curves are rectifiable. If $\Gamma$ is convex on $E_{m}$ and $m$ is $o d d$, then the arc $\Gamma$ can never close. In even-dimensional spaces $E_{2 n}$ we may well have closed curves convex on $E_{2 n}$ as seen by the example of the special curve

$$
\begin{aligned}
C_{0}: x_{1}=\cos t, x_{3} & =\frac{1}{2} \cos 2 t, \cdots, x_{2 n-1}= \\
x_{2}=\sin t, x_{4}=\frac{1}{2} \sin 2 t, \cdots, x_{2 n}= & \frac{1}{n} \sin n t, \\
& (0 \leqq t \leqq 2 \pi) .
\end{aligned}
$$


That $C_{0}$ spans $E_{2 n}$ is clear and equally so that $C_{0}$ is convex in $E_{2 n}$ : If $l\left(x_{i}\right)$ is a linear function and if we substitute the $x_{i}$ from (2.7), then $l\left(x_{i}\right)$ becomes a real trigonometric polynomial of order $n$ which is known to change sign within a period at most $2 n$ times.

Let

$$
C: x_{i}=x_{i}(t) \quad(i=1, \cdots, 2 n ; 0 \leqq t \leqq 2 \pi),
$$

be a closed curve in $E_{2 n}$ defined by continuous functions of period $2 \pi$. $C$ is found to be convex on $E_{2 n}$ provided the above restriction on the signs of the determinants (2.6) (with $m=2 n$ ) holds for parameter values restricted by the inequalities $t_{0}<t_{1}<\cdots<t_{n}<t_{0}+2 \pi$. This criterion leads naturally to a useful expression for the $2 n$-dimensional volume $V=V(K)$ of the convex hull $K=K(C)$ of the curve $C$. Indeed, assuming the $x_{i}(t)$ to be absolutely continuous, as is the case if $t$ is proportional to the arc-length, then $V$ can be expressed by the following Lebesgue integral

$$
\begin{array}{r}
V=\frac{\epsilon}{n !(2 n) !} \int_{0}^{2 \pi} \cdots \int_{0}^{2 \pi} \operatorname{det} \| x_{i}\left(t_{1}\right), x_{i}{ }^{\prime}\left(t_{1}\right), x_{i}\left(t_{2}\right), x_{i}{ }^{\prime}\left(t_{2}\right), \cdots, \\
x_{i}\left(t_{n}\right), x_{i}{ }^{\prime}\left(t_{n}\right) \| d t_{1} \cdots d t_{n},
\end{array}
$$

where $\epsilon=1$, or -1 , depending on the common sign of the determinants (2.6). This expression for $V$, which reduces to the classical areaintegral if $n=1$, allows one to generalize, by A. Hurwitz's method of Fourier series [3], the classical isoperimetric inequality to closed curves in $E_{2 n}$ : If the closed curve $C$ is convex in $E_{2 n}$, of length $L$, then the following inequality holds:

$$
L^{2 n} \geqq(2 \pi n)^{n} n !(2 n) ! V,
$$

with the equality sign if and only if $C$ is similar to the curve $C_{0}$, defined by (2.7), or to its reflexion (see [56]). If $n=1,(2.9)$ reduces to the well known inequality $L^{2} \geqq 4 \pi V$ for convex curves in $E_{2}, V$ being the area enclosed by $C$, and where equality holds only if $C$ is a circle.

It is to be expected that this new approach to curves convex in $E_{m}$ will be fruitful and work in collaboration with T. S. Motzkin is in progress. In concluding it should be pointed out that the notion of convexity of curves is also related to the Chebyshev systems of functions (Kellogg $[9 ; 10 ; 11]$, S. Bernstein [16], Laasonen [42]) and to the systems satisfying Descartes' rule of signs (Pólya-Szegö [14]). Especially close is Pólya's discussion [13] of linear homogeneous differential equations. Indeed, the notion of a curve convex on $E_{m}$ being affine invariant, such differential equations would seem to be the appropriate tool. 


\section{VARIATION-DIMINISHING INTEGRAL TRANSFORMATIONS}

3.1. Pólya's work of 1915. An entire function $\Psi(s)$ may obviously be represented as a limit of a sequence of polynomials, uniformly convergent in every bounded domain of the complex plane: The sections of its expansion in powers of $s$ have this property. Laguerre [1] and Pólya [5] have investigated the following problem: Which entire $\Psi(s)(\not \equiv 0)$ are limits of a sequence of real polynomials having only real zeros? They found this to be the case if and only if $\Psi(s)$ is of the form

$$
\begin{aligned}
\Psi(s)=C e^{-\gamma s^{2}+\delta_{s}} s^{m} & \prod_{1}^{\infty}\left(1+\delta_{\nu} s\right) e^{-\delta_{\nu} s} \\
(C & \left.\gtrless 0, \gamma \geqq 0, \delta, \delta_{\nu} \text { real, } \sum \delta_{\nu}<\infty\right) .
\end{aligned}
$$

They also showed that the subclass of those functions $\Phi(s)$ which are limits of polynomials with only real zeros and non-negative coefficients is characterized by the representation

$$
\Phi(s)=C e^{\gamma s} s^{m} \prod_{1}^{\infty}\left(1+\delta_{\nu} s\right) \quad\left(C>0, \gamma \geqq 0, \delta_{\nu} \geqq 0, \sum \delta_{\nu}<\infty\right) .
$$

See also Szász [28]. For an extension of these results to entire functions of $n$ variables see Motzkin-Schoenberg [50].

Pólya and Schur [7] have described these classes in terms of their Taylor expansions: A formal real power series

$$
a_{0}+\left(a_{1} / 1 !\right) s+\left(a_{2} / 2 !\right) s^{2}+\cdots
$$

is the expansion of a function of the form (3.1) if and only if the polynomials

$$
P_{n}(x)=a_{0}+\left(\begin{array}{l}
n \\
1
\end{array}\right) a_{1} x+\cdots+a_{n} x^{n} \quad(n=1,2, \cdots)
$$

have only real zeros; also that it is the expansion of a function of the form (3.2) if and only if the polynomials (3.3) have only real zeros and non-negative coefficients.

In [8], Pólya considered the subclass

$$
\begin{aligned}
\Psi(s)=C e^{-\gamma s^{2}+\delta s} \prod_{1}^{\infty}\left(1+\delta_{\nu} s\right) e^{-\delta_{\nu} s} \\
\quad\left(C>0, \gamma \geqq 0, \delta, \delta_{\nu} \text { real, } 0<\gamma+\sum \delta_{\nu}^{2}<\infty\right),
\end{aligned}
$$

of those functions of the class (3.1), with $\Psi(0)>0$, which are not 
exponentials $C \exp \{\delta s\}$, and investigated the power series expansions of their reciprocals

$$
\frac{1}{\Psi(s)}=\sum_{0}^{\infty} \frac{(-1)^{\nu} \mu_{\nu}}{\nu !} s^{\nu} .
$$

He discovered the following properties:

1. The Hankel determinants

$$
\left|\begin{array}{cccc}
\mu_{0} & \mu_{1} & \cdots & \mu_{n} \\
\mu_{1} & \mu_{2} & \cdots & \mu_{n+1} \\
\vdots & & & \\
\mu_{n} & \mu_{n+1} & \cdots & \mu_{2 n}
\end{array}\right|
$$

2. If $f(x)$ is a real polynomial, then

$$
g(x)=\sum_{0}^{\infty} \frac{(-1)^{\nu} \mu_{\nu}}{\nu !} f^{(\nu)}(x)=\frac{1}{\Psi(D)} f(x)
$$

is a polynomial having no more real zeros than $f(x)$. Conversely, if a real formal power series $\sum(-1)^{\nu} \mu_{\nu} s^{\nu} / \nu !\left(\mu_{0}>0\right)$ is such that $g(x)$, defined by (3.7), has never more zeros than the arbitrary polynomial $f(x)$, then it is the expansion (3.5) of the reciprocal of a function $\Psi(s)$ of the form (3.4).

In 1920 Hamburger [12], using his then recent solution of the moment problem bearing his name, concluded from (3.6) that the coefficients $\mu_{\nu}$, of (3.5), are the moments of a nondecreasing function $\psi(x)$ and that (3.5) may be represented as a Laplace-Stieltjes integral

$$
\frac{1}{\Psi(s)}=\int_{-\infty}^{\infty} e^{-x s} d \psi(x)
$$

within the vertical strip of regularity of the left-hand function, containing the origin of the $s$-plane. More recently Hirschman-Widder [40] and Schoenberg $[34 ; 55]$ have established a representation of (3.5) in terms of a bilateral Laplace integral

$$
\frac{1}{\Psi(s)}=\int_{-\infty}^{\infty} e^{-x s} \Lambda(x) d x
$$

Here $\Lambda(x)$ is a frequency function, ${ }^{7}$ i.e. $\Lambda(x) \geqq 0$, which may be repre-

${ }^{7}$ Widder [35] interprets $\Lambda(x-y)$ as the Green's function of a differential system for the whole real axis. For a thorough investigation of differential systems in a finite range whose Green's functions are totally positive (Kellogg kernels) see GantmakherKrein [44]. 
sented by the familiar inversion formula

$$
\Lambda(x)=\frac{1}{2 \pi i} \int_{-i \infty}^{i \infty} \frac{e^{x s}}{\Psi(s)} d s \quad(-\infty<x<\infty),
$$

where the integral is defined as usual as a principal value.

Since

$$
\mu_{\nu}=\int_{-\infty}^{\infty} x^{\nu} \Lambda(x) d x
$$

we may now write the differential operation (3.7) as an integral transformation. Indeed, if $f(x)$ is a polynomial, we find

$$
\begin{aligned}
\int_{-\infty}^{\infty} \Lambda(t) f(x-t) d t & =\int_{-\infty}^{\infty} \Lambda(t)\left\{\sum_{0}^{\infty} \frac{1}{\nu !} f^{(\nu)}(x)(-t)^{\nu}\right\} d t \\
& =\sum_{0}^{\infty} \frac{(-1)^{\nu} \mu_{\nu}}{\nu !} f^{(\nu)}(x)=g(x)
\end{aligned}
$$

or

$$
g(x)=\int_{-\infty}^{\infty} \Lambda(x-t) f(t) d t
$$

Hence for polynomials, (3.10) being equivalent to (3.7), this integral transformation is inverted by

$$
f(x)=\Psi(D) g(x)
$$

where the operator $\Psi(D)$ is to be applied after its expansion in powers of $D$. This theory goes back to Pólya's paper [8] of 1915, except for the integral representations (3.8) and (3.10), the transformation (3.10), for polynomials, appearing in Pólya's paper in terms of the equivalent differential operation (3.7).

3.2. Pólya frequency functions. The determining functions $\Lambda(x)$, of (3.8), may be described in terms of the following definition: A frequency function $\Lambda(x)$, i.e. a measurable non-negative function such that

$$
0<\int_{-\infty}^{\infty} \Lambda(x) d x<\infty
$$

is said to be a Pólya frequency function provided that for any two sets of numbers

$$
x_{1}<x_{2}<\cdots<x_{n}, \quad t_{1}<t_{2}<\cdots<t_{n} \quad(n=1,2, \cdots)
$$


we have the inequality

$$
\operatorname{det}\left\|\Lambda\left(x_{i}-t_{j}\right)\right\|_{1, n} \geqq 0 .
$$

In terms of this definition, the following theorem holds: If $\Psi(s)$ is of the form (3.4), then its reciprocal may be represented in the form (3.8) within the vertical strip of regularity of $1 / \Psi$ which contains the origin, and where $\Lambda(x)$ is a Pólya frequency function. Conversely, given a Pólya frequency function $\Lambda(x)$, then its Laplace transform (3.8) converges within such a vertical strip and represents the reciprocal of an entire function of the form (3.4). See Schoenberg [34; 55]; also Wintner [23, Theorem 1].

Similar results concerning the ordinary Laplace transform are connected with entire functions of the form

$$
\begin{aligned}
\Phi(s) & =C e^{\gamma s} \prod_{1}^{\infty}\left(1+\delta_{\nu} s\right) \\
& \left(C>0, \gamma \geqq 0, \delta_{\nu} \geqq 0,0<\gamma+\sum \delta_{\nu}<\infty\right) .
\end{aligned}
$$

These functions forming a subclass of the class of functions (3.4), the previous theorem applies to their reciprocals. However, the function $\Psi(s)$ of (3.8) is of the special form (3.14) if and only if the Pólya frequency function $\Lambda(x)$, of (3.8), vanishes for all negative values of $x$ (see $[34 ; 55])$.

A related question is the following: The meromorphic function on the left-hand side of (3.8) has in general several vertical strips of regularity separated by vertical lines through its poles. What is the nature of the Laplace integral representations of $1 / \Psi$ in the strips which do not contain the origin? An answer requires the following definition: A real measurable function $\Lambda(x)(-\infty<x<\infty)$ is said to be totally positive provided it satisfies the following three conditions: 1. (3.12) implies (3.13), 2. $\Lambda(x)$ is different from zero for at least two distinct values of $x, 3 . \Lambda(x) \not \equiv \exp \{a x+b\}$. It is clear that the Pólya frequency functions are totally positive. Moreover, it can be shown that every totally positive function turns into a Pólya frequency function, upon multiplication by appropriate exponentials. As a result the above stated correspondence between functions $\Psi(s)$ and Pólya frequency functions now extends to a one-to-one correspondence between functions $\Psi(s)$ and the totally positive functions $\Lambda(x)$, by the relation (3.8) with the sign changed if necessary, in vertical strips of regularity for $1 / \Psi$ which are no longer required to contain the origin. In particular, a given $1 / \Psi(s)$ allows a representation (3.8) in each of its strips of regularity with a different totally positive $\Lambda(x)$ corresponding to each of the strips (see $[34 ; 55]$ ). 
As particular examples of Pólya frequency functions we mention

$$
\Lambda_{1}(x)=e^{-x^{2}}, \quad \Lambda_{2}(x)=e^{-x-e^{-x}}, \quad \Lambda_{3}(x)=\frac{1}{\cosh x},
$$

whose respective transforms $\pi^{1 / 2} e^{s^{2} / 4}, \Gamma(s+1), \pi / \cos (\pi s / 2)$ are seen to be reciprocals of functions of the form (3.4). As a nontrivial example of Pólya frequency functions vanishing for $x<0$ we mention

$$
\Lambda_{4}(x)= \begin{cases}\sum_{-\infty}^{\infty}(-1)^{\nu+1} \nu^{2} e^{-x \nu^{2}} & \text { if } x>0, \\ 0 & \text { if } x \leqq 0,\end{cases}
$$

whose transform $\prod_{1}^{\infty}\left(1+s^{2} / \nu^{2}\right)^{-1}$ is indeed the reciprocal of a function of the form (3.14). Its cumulative distribution function

$$
\int_{-\infty}^{x} \Lambda_{4}(x) d x= \begin{cases}\sum_{-\infty}^{\infty}(-1)^{v} e^{-x \nu^{2}} & \text { if } x>0, \\ 0 & \text { if } x \leqq 0\end{cases}
$$

is the subject of the Kolmogorov-Smirnov limit theorem (see Feller [36]).

Given a Pólya frequency function $\Lambda(x)$ in terms of its transform (3.8) and two sets of numbers (3.12), Schoenberg-Whitney [43; 57] have answered the question as to when the determinant (3.13) is actually positive. For a discussion of the differentiability properties of Pólya frequency functions see [55, Corollary 2], Widder [35, Theorems 7 and 12], Hirschman-Widder [40]. For a new characterization of Pólya frequency functions as the only possible limits of so-called spline frequency functions see Curry-Schoenberg [33 ]. Concerning the interesting property of Pólya frequency functions of being "bell-shaped" see Hirschman-Widder [40], Schoenberg [34], and a related note [45] by Hirschman.

3.3. Smoothing properties of Pólya frequency functions. The remarkable smoothing property of the transformation (3.7), or (3.10), to the effect that the polynomial $g(x)$ has no more real zeros than $f(x)$, suggested the problem of finding the most general transformation of the form (3.10) which is variation-diminishing. By this we mean, obviously enough, the following: Let $v(f)$ (the number of changes of sign of $f(x)$ ) denote the supremum of the number of changes of sign in the sequence $f\left(x_{1}\right), \cdots, f\left(x_{n}\right)$, where $-\infty<x_{1}$ $<\cdots<x_{n}<+\infty, n=2,3, \cdots$. Let $\Lambda(x)$ be summable, not 
$=0$ p.p., and such that, for every bounded $f(x),(3.10)$ should imply the inequality

$$
v(g) \leqq v(f) .
$$

It turns out that this is the case if and only if $\Lambda(x)$ or $-\Lambda(x)$ is a Pólya frequency function. The variation-diminishing property of (3.10) therefore characterizes the Pólya frequency functions. For a somewhat stronger result in terms of Stieltjes integrals see Schoenberg [46]. The author's old theorem [19] to the effect that the linear transformation (2.1) is variation-diminishing if the matrix $A$ is totally positive is the origin of the peculiar property (3.13) of Pólya frequency functions. Indeed, (3.10) may be regarded as a linear transformation defined by the matrix $\Lambda(x-t)$, while (3.13) expresses its total positivity.

3.4. Inversion and representation problems. The prototype of the functional transformations to be now discussed is the Laplace transformation

$$
\gamma(z)=\int_{0}^{\infty} e^{-z u} \phi(u) d u .
$$

Well known results are its inversion by means of the Post-Widder inversion operator (Widder [32, Chap. VII]) and the BernsteinWidder theorem which characterizes the functions representable in the form

$$
\gamma(z)=\int_{0}^{\infty} e^{-z u} d \alpha(u) \quad(d \alpha(u) \geqq 0)
$$

by the condition of complete monotoneity [32, Chap. VII, \$14]. Following Widder we now set $u=\exp (t), z=\exp (-x)$, whereupon (3.16) changes into

$$
g(x)=\int_{-\infty}^{\infty} \Lambda_{2}(x-t) f(t) d t
$$

where $g(x)=\gamma\left(e^{-x}\right) e^{-x}, f(t)=\phi\left(e^{t}\right)$, while $\Lambda_{2}(x)=\exp \left(-x-e^{-x}\right)$ is one of the Pólya frequency functions (3.15). The Laplace transformation (3.16) is thus seen to be equivalent to a special instance of the convolution transformation (3.10) ${ }^{8}$ For wide classes of Pólya fre-

8 This explains, in view of Article 3.3, the variation-diminishing properties of the Laplace transformation discovered by Laguerre and Pólya. See Pólya-Szegö [14, Problem 80, p. 50]. 
quency functions $\Lambda(x)$, Hirschman and Widder have extended the above results concerning the Laplace transformation to the general transformation

$$
g(x)=\int_{-\infty}^{\infty} \Lambda(x-t) f(t) d t,
$$

obtaining an inversion theory (Widder $[35 ; 52 ; 53]$, HirschmanWidder $[37 ; 40]$ ) and a representation theory (Hirschmann-Widder [41]). Their results include as special cases earlier work by R. P. Boas and H. Pollard on the so-called Stieltjes transformation (which again may be reduced to (3.18) if $\left.\Lambda(x)=\Lambda_{3}(x)=1 / \cosh x\right)$ and its iterates. In case $f(x)$ is a polynomial we have seen that (3.18) is inverted by the differential operation (3.11). However, for arbitrary $f(x)$ the relation (3.11) is meaningless.

In order to illustrate the Hirschman-Widder inversion theory for (3.18) and to see how it goes beyond Pólya's formal inversion by (3.11), let us consider briefly the following special case discussed by Widder, The inversion of a generalized Laplace transform, Proc. Nat. Acad. Sci. U.S.A. vol. 33 (1947) pp. 295-297: Let $\Lambda(x)$ be a Pólya frequency function defined by the relations (3.8) and (3.4), with $\gamma=0$ and all $\delta_{\nu} \neq 0$. If the given function $f(x)$ is continuous and summable $(f \in C \cdot L)$, then (3.18) is inverted by the relation

$$
f(x)=\lim _{n \rightarrow \infty} \prod_{\nu=1}^{n}\left(1+\delta_{\nu} D\right) g\left(x-\sum_{1}^{n} \delta_{\nu}\right) \quad(-\infty<x<\infty) .
$$

A proof follows readily from the relation

$$
(1+s \delta) e^{-\delta s} / \Psi(s)=\int_{-\infty}^{\infty} e^{-s x}(1+\delta D) \Lambda(x-\delta) d x,
$$

where $\delta$ is a real constant, and which follows from (3.8) by integration by parts. Applying the transformation (3.20) to (3.8), $n$ times in succession with $\delta=\delta_{1}, \delta_{2}, \cdots, \delta_{n}$, we obtain the relation

$$
\prod_{\nu=n+1}^{\infty} \frac{e^{\delta_{\nu} s}}{1+\delta_{\nu} S}=\int_{-\infty}^{\infty} e^{-x s} \Lambda_{n}(x) d x,
$$

where $\Lambda_{n}(x)$ is evidently a Pólya frequency function explicitly given by

$$
\Lambda_{n}(x)=\prod_{\nu=1}^{n}\left(1+\delta_{\nu} D\right) \Lambda\left(x-\sum_{1}^{n} \delta_{\mu}\right)
$$


If we now operate with

$$
\prod_{\nu=1}^{n}\left(1+\delta_{\nu} D\right) e^{-\delta_{\nu} D}
$$

on both sides of the relation (3.18), we obtain, by (3.22),

$$
f_{n}(x) \equiv \prod_{1}^{n}\left(1+\delta_{\nu} D\right) g\left(x-\sum_{1}^{n} \delta_{\mu}\right)=\int_{-\infty}^{\infty} \Lambda_{n}(x-t) f(t) d t
$$

and we are to show that

$$
f_{n}(x)=\int_{-\infty}^{\infty} \Lambda_{n}(x-t) f(t) d t \rightarrow f(x), \quad \text { as } n \rightarrow \infty .
$$

This follows from the fact that (3.23) is a singular integral satisfying the classical conditions of Jordan:

$$
\begin{array}{cr}
\int_{-\infty}^{\infty} \Lambda_{n}(x) d x=1 & \text { for all } n, \\
\lim _{n \rightarrow \infty} \int_{|x| \geqq \delta} \Lambda_{n}(x) d x=0, & \text { for every } \delta>0 .
\end{array}
$$

Indeed, on expanding both sides of (3.21) in powers of $s$ and comparing coefficients we find

$$
\int_{-\infty}^{\infty} \Lambda_{n}(x) d x=1, \quad \int_{-\infty}^{\infty} x^{2} \Lambda_{n}(x) d x=\sum_{n+1}^{\infty} \delta_{\nu}^{2}=\sigma_{n}^{2} .
$$

The fact that the variance $\sigma_{n}^{2}$ converges to zero, as $n \rightarrow \infty$, implies the property (ii) by a familiar Chebyshev argument. See Widder, loc. cit., where it is shown that the Post-Widder inversion operator for the Laplace transformation (3.16) is a special case of (3.19).

See Widder $[\mathbf{5 2} ; \mathbf{5 3}]$ for the particularly interesting case of $\Lambda(x)=\Lambda_{1}(x)=\exp \left(-x^{2}\right)$ when (3.18) becomes the Weierstrass transform.

\section{VARIATION DIMINISHING SEQUENCE TRANSFORMATIONS}

\subsection{Totally positive sequences. The sequence transformation}

$$
y_{n}=\sum_{\nu=-\infty}^{\infty} a_{n-\nu} x_{\nu}
$$

gives rise to a theory in some respects analogous to the theory of $\$ 3$. Assuming that $\sum\left|a_{\nu}\right|<\infty$, we say that the transformation (4.1) 
is variation-diminishing, provided that for every bounded sequence $\left\{x_{n}\right\},(4.1)$ implies the inequality

$$
v(y) \leqq v(x) .
$$

Here, as before, $v(x)$ and $v(y)$ denote the numbers of changes of sign in their respective sequences and may well be infinite. Since $x_{0}=1$, $x_{n}=0$ (if $n \neq 0$ ) implies that $y_{n}=a_{n}$, for all $n$, we see that (4.2) implies that $v\left(a_{n}\right)=0$. We may therefore assume all $a_{n}$ non-negative.

A sequence $\left\{a_{n}\right\}$ is called totally positive provided that the matrix

$$
A=\left\|a_{i-j}\right\| \quad(-\infty<i, j<\infty),
$$

is totally positive, i.e. has only non-negative minors, and that $\left\{a_{n}\right\}$ is not a geometric progression of the form $a_{n}=C r^{n}(C \geqq 0, r>0)$. We say that the sequence $\left\{a_{n}\right\}$ is totally positive normalized provided $\left\{a_{n}\right\}$ is totally positive and the series $\sum a_{n}$ converges. The totally positive normalized sequences are the discrete analogues of the Pólya frequency functions of $\$ 3$. A restricted kind of total positivity ( $k$-times positive sequences) was discussed as early as 1912 by M. Fekete [4].

We saw in $\$ 2$ that the transformation (2.1) is variation-diminishing if its matrix $\left\|a_{i k}\right\|$ is totally positive. In our case of the convolution transformation (4.1) this condition is not only sufficient but also necessary as stated by the following theorem: The transformation (4.1) is variation-diminishing if and only if the sequence $\left\{a_{n}\right\}$ is totally positive normalized (see [38]).

4.2. Generation of totally positive sequences: Edrei's theorem. In view of the last theorem, the problem of constructing variationdiminishing transformations (4.1) is equivalent to the problem of constructing sequences $\left\{a_{n}\right\}$ which are totally positive and normalized. A first step in this direction was made in [38] where the following was proved: If

$$
F(z)=C e^{a z+b z^{-1}} z^{m} \frac{\prod_{1}^{\infty}\left(1+\alpha_{\nu} z\right) \prod_{1}^{\infty}\left(1+\beta_{\nu} z^{-1}\right)}{\prod_{1}^{\infty}\left(1-\gamma_{\nu} z\right) \prod_{1}^{\infty}\left(1-\delta_{\nu} z^{-1}\right)}
$$

is a meromorphic function in the ring $0<|z|<\infty$, such that its constants satisfy the conditions

$$
\begin{aligned}
& C>0, a \geqq 0, b \geqq 0, m \text { integer, } \alpha_{\nu} \geqq 0, \beta_{\nu} \geqq 0, \\
& 0 \leqq \gamma_{\nu}<1,0 \leqq \delta_{\nu}<1, \sum\left(\alpha_{\nu}+\beta_{\nu}+\gamma_{\nu}+\delta_{\nu}\right)<\infty,
\end{aligned}
$$


and if we expand $F(z)$ in a Laurent series

$$
F(z)=\sum_{-\infty}^{\infty} a_{n} z^{n} \quad\left(r<|z|<r^{-1}\right)
$$

in the ring containing the unit circle inside, then the sequence $\left\{a_{n}\right\}$ so generated is totally positive normalized. ${ }^{9}$

This was easily established, the real difficulties arising as soon as we raise the following inverse problem: Given a totally positive normalized sequence $\left\{a_{n}\right\}$, it is not hard to show that the corresponding Laurent series (4.6) converges in an open ring containing the unit circle and defining an analytic function $F(z)$. Is it true, then, that $F(z)$ is meromorphic in $0<|z|<\infty$ and of the form (4.4), (4.5)?

Let us look at some examples. The following remark is obvious: If $\Lambda(x)$ is a Pólya frequency function (Article 3.2) then $a_{n}=\Lambda(n)$ $(-\infty<n<\infty)$ is a totally positive sequence which is normalized; for, indeed, $\left\|a_{i-j}\right\|=\|\Lambda(i-j)\|$ is a "submatrix" of $\Lambda(x-t)$. Using the functions (3.15) we now obtain sequences $\left\{a_{n}\right\}$ for exploratory purposes. Choosing $\Lambda(x)=\exp \left(-h x^{2}\right)$, where $h$ is a positive constant and setting $q=\exp (-h)$, we obtain the sequence $a_{n}=\Lambda(n)=q^{n^{2}}$ which is totally positive and normalized. That the corresponding generating function (4.6) is of the form (4.4), (4.5) is shown by the famous Jacobian identity

$$
\sum_{-\infty}^{\infty} q^{n^{2}} z^{n}=C_{1} \prod_{n=0}^{\infty}\left(1+q^{2 n+1} z\right)\left(1+q^{2 n+1} z^{-1}\right) \quad(0<|z|<\infty),
$$

where $C_{1}$ does not depend on $z$. For the same reason (see (3.15)) also the sequence

$$
a_{n}=\Lambda_{3}(h n)=1 / \cosh (h n)
$$

is totally positive normalized. The following identity which is equivalent to the Fourier expansion of the elliptic function $\operatorname{dn} u$ :

$$
\sum_{-\infty}^{\infty} \frac{1}{\cosh (h n)} z^{n}=C_{2} \frac{\prod_{0}^{\infty}\left(1+q^{2 n+1} z\right)\left(1+q^{2 n+1} z^{-1}\right)}{\prod_{0}^{\infty}\left(1-q^{2 n+1} z\right)\left(1-q^{2 n+1} z^{-1}\right)}\left(q<|z|<q^{-1}\right)
$$

${ }^{9}$ In Rademacher-Schoenberg [30, pp. 156-159], the function $-1 / J_{0}\left((13 z)^{1 / 2}\right)$ is expanded, as a numerical illustration, in a Laurent series $\sum \omega_{n} z^{n}$ in a ring between its first and second pole. The extremely smooth behavior of the coefficients $\omega_{n}$ there obtained is explained by the fact that the sequence $\left\{\omega_{n}\right\}$ is totally positive normalized, in view of the result just stated. 
shows that its generating function is of the form (4.4), (4.5).

It was conjectured in [38] that the answer to the question is affirmative. This was recently established by A. Edrei [62] who proved the following theorem: If $\left\{a_{n}\right\}$ is a sequence which is totally positive and normalized, then its generating function (4.6) is necessarily of the form (4.4), (4.5). ${ }^{10}$

Three noteworthy special cases, stated with their converses, are as follows:

1. If the power series

$$
\sum_{0}^{\infty} a_{n} z^{n} \quad\left(a_{0}=1\right)
$$

converges for all $z$, then it represents a function of the form

$$
e^{\gamma z} \prod_{1}^{\infty}\left(1+\delta_{\nu} z\right) \quad\left(\gamma \geqq 0, \delta_{\nu} \geqq 0, \sum \delta_{\nu}<\infty\right)
$$

if and only if the matrix

$$
A=\left\|\begin{array}{llll}
a_{0} & 0 & 0 & \cdots \\
a_{1} & a_{0} & 0 & \cdots \\
a_{2} & a_{1} & a_{0} & \cdots \\
\vdots & \vdots & \vdots &
\end{array}\right\|
$$

is totally positive.

2. If the Laurent series

$$
\sum_{-\infty}^{\infty} a_{n} z^{n}
$$

converges for all $z \neq 0$, then it represents a function of the form

$$
C e^{a z+b z^{-1}} z^{m} \prod_{1}^{\infty}\left(1+\alpha_{\nu} z\right) \prod_{1}^{\infty}\left(1+\beta_{\nu} z^{-1}\right)
$$

$$
\left(C \geqq 0, a \geqq 0, b \geqq 0, m \text { integer, } \alpha_{\nu} \geqq 0, \beta_{\nu} \geqq 0, \sum\left(\alpha_{\nu}+\beta_{\nu}\right)<\infty\right)
$$

${ }^{10}$ Notice the interesting symmetry of (4.4) with respect to reciprocation: Also $1 / F(-z)$ is of the form (4.4). An easy corollary of Edrei's theorem is as follows: If the identity $\left(\sum_{-\infty}^{\infty} a_{n} z^{n}\right)\left(\sum_{-\infty}^{\infty}(-1)^{n} b_{n} z^{n}\right)=1$ holds in a ring $r_{1}<|z|<r_{2}$, and if $\left\{a_{n}\right\}$ is totally positive, then either $\left\{b_{n}\right\}$ or else $\left\{-b_{n}\right\}$ is a totally positive sequence. As it happens, this proposition is actually a lemma in Edrei's proof of his theorem.

It should also be remarked that in analogy with the situation of $\$ 3$, the Laurent expansions of (4.4) in every ring of regularity of $F(z)$ will generate, up to a common negative sign, totally positive sequences. 
if and only if the sequence $\left\{a_{n}\right\}$ is totally positive.

3. If the symmetric Laurent series

$$
\sum_{-\infty}^{\infty} a_{n} z^{n} \quad\left(a_{n}=a_{-n}\right)
$$

converges for all $z \neq 0$, then it represents a function of the form

$$
C e^{a\left(z+z^{-1}\right)} \prod_{1}^{\infty}\left(1+\alpha_{\nu} z\right)\left(1+\alpha_{\nu} z^{-1}\right)
$$

$$
\left(C \geqq 0, a \geqq 0,1 \geqq \alpha_{1} \geqq \alpha_{2} \geqq \cdots \geqq \alpha_{n} \geqq \cdots \geqq 0, \sum \alpha_{\nu}<\infty\right)
$$

if and only if the sequence $\left\{a_{n}\right\}$ is totally positive.

For a different characterization of the power series expansion of entire functions of the form (4.8) see J. Grommer [6, pp. 157-158]. A third characterization of the same expansion in terms of the zeros of the polynomials (3.3) was stated in Article 3.1. Edrei's general theorem should be regarded as a notable contribution to the general type of problem initiated by Hadamard in his classical dissertation [2]. Of course, the condition of Edrei's theorem that the sequence $\left\{a_{n}\right\}$ be totally positive is very strong, but then (4.4), (4.5) show the precise nature of the information on $F(z)$ which the theorem furnishes. Moreover, the condition is not only sufficient but also necessary.

Let us briefly review the work leading up to Edrei's theorem. Aissen, Schoenberg, and Whitney [59] investigated totally positive sequences $\left\{a_{n}\right\}$ with the property that $a_{n}=0$ if $n<0, a_{0}=1$, and showed that their generating function is necessarily of the form

$$
\sum^{\infty} a_{n} z^{n}=e^{g(z)} \frac{\prod_{1}^{\infty}\left(1+\alpha_{\nu} z\right)}{\prod_{1}^{\infty}\left(1-\gamma_{\nu} z\right)}
$$

where $\alpha_{\nu} \geqq 0, \gamma_{\nu} \geqq 0, \sum\left(\alpha_{\nu}+\gamma_{\nu}\right)<\infty$, and where $g(z)$ is entire and such that the exponential factor $\exp \{g(z)\}$, if expanded in powers of $z$, will also generate a totally positive sequence. Combining this result with a refinement of Picard's theorem due to R. Nevannlina, Edrei [60] showed that necessarily $g(z)=\gamma z(\gamma \geqq 0)$, thereby proving his theorem for the case when (4.6) reduces to a power series. A different approach to this case was given by Edrei in [61]. For the general case see Edrei [62].

4.3. An application of Edrei's theorem. With the problem to be 
now discussed we return to the stability of smoothing formulae which is the theme of $\$ 1$. In 1946 the author constructed certain classes of analytic approximation formulae to given equidistant ordinates $\left\{y_{n}\right\}$ $(-\infty<n<\infty)$, which we need not describe here in detail; they are of the "cardinal" type

$$
f(x)=\sum_{\nu=-\infty}^{\infty} y_{\nu} L(x-\nu),
$$

$L(x)$ being a certain even entire function, accurate tables of values for $L(x)$ and its derivatives being available. Moreover, if $y_{\nu}=P(\nu)$, where $P(x)$ is a polynomial of degree $2 k-1$ ( $k$ being a certain fixed number), then $f(x) \equiv P(x)$. If we now let $x=n$ be an integer, we are faced with the natural question whether the sequence transformation

$$
f(n)=\sum_{\nu=-\infty}^{\infty} L(n-\nu) y_{\nu}
$$

satisfies our stability (or smoothing) condition (1.6) (see Schoenberg [31, Part B, pp. 131-133]). This question was there shown to depend on a conjecture which the author was unable to prove at the time. The conjecture there formulated is an easy corollary of a theorem to be stated and proved below by means of Edrei's theorem.

We start with the "rectangular" frequency function

$$
M_{1}(x)=\left\{\begin{array}{lll}
1 & \text { if } & |x| \leqq 1 / 2 \\
0 & \text { if } & |x|>1 / 2
\end{array}\right.
$$

having the transform

$$
\int_{-\infty}^{\infty} e^{-x \cdot} M_{1}(x) d x=\frac{2 \sinh (s / 2)}{s}
$$

and the normal frequency function

$$
M_{0}(x, t)=\frac{1}{(\pi t)^{1 / 2}} e^{-x^{2} / t}
$$

of transform

$$
\int_{-\infty}^{\infty} e^{-x s} M_{0}(x, t) d x=e^{t s^{2} / 4} .
$$

If we convolute $M_{1}(x)$ with itself $k-1$ times and convolute the result with $M_{0}(x, t)$, we obtain a distribution function $M_{k}(x, t)$ whose transform, by (4.12) and (4.14), is evidently 


$$
\int_{-\infty}^{\infty} e^{-x s} M_{k}(x, t) d x=e^{t s^{2} / 4}\left(\frac{2 \sinh s / 2}{s}\right)^{k}
$$

The theorem to be proved is as follows: The Laurent series

$$
F(z)=\sum_{-\infty}^{\infty} M_{k}(n, t) z^{n} \quad(0<|z|<\infty)
$$

admits a product representation

$$
F(z)=C \cdot \prod_{\nu=1}^{\infty}\left(1+\alpha_{\nu} z\right)\left(1+\alpha_{\nu} z^{-1}\right)
$$

where the $\alpha_{\nu}=\alpha_{\nu}(t)$ are real and satisfy the conditions $0<\alpha_{\nu}<1$, $\sum \alpha_{\nu}<\infty$, while $C=\Pi\left(1+\alpha_{\nu}\right)^{-2}$.

REMARKs." 1 . The conclusion (4.17) "implies" that the sequence $a_{n}=M_{k}(n, t)$ is totally positive. This, naturally, will turn out to be the case. However, if $k>0$, the transform (4.15), of $M_{k}(x, t)$, has zeros. It is therefore not of the form (3.8) and we conclude that $M_{k}(x, t)$ is not a Pólya frequency function. We can therefore not apply the convenient argument used in Article 4.2 to show that the sequence $M_{k}(n, t)$ is totally positive.

2. The argument is of course valid if $k=0$ since $M_{0}(x, t)$ is the normal function (4.13). In this case our conclusion (4.17) is evidently true since it reduces to Jacobi's identity (4.7). If $k>0$, the representation (4.17) may be regarded as a generalization of Jacobi's relation.

3. Quite recently, J. Berghuis [54] succeeded in proving (4.17) for the two values $k=1$ and $k=2$ by standard methods of the theory of functions.

A general proof of (4.17) is as follows: We define the successive integrals of $(4.13)$ by the relations

and

$$
g_{0}(x)=M_{0}(x, t)
$$

$$
g_{\nu}(x)=\int_{-\infty}^{x} g_{\nu-1}(x) d x \quad(\nu=1,2, \cdots) .
$$

The Laplace transform of $g_{k}(x)$ is found from (4.14), by successive

${ }^{11}$ The conjecture stated in [31, Part B, pp. 131-132] is as follows: The reciprocal of the function $F\left(e^{i u}\right)$ admits a representation $\left(^{*}\right) 1 / F\left(e^{i u}\right)=\sum_{0}^{\infty} C_{2 n}(t)(2 \sin u / 2)^{2 n}$ whose coefficients are positive and which converges for all real $u$. This is implied by (4.17) as follows: $F\left(e^{i u}\right)=C \prod\left(1+\alpha_{\nu}{ }^{2}+2 \alpha_{\nu} \cos u\right)=C \prod\left\{\left(1+\alpha_{\nu}\right)^{2}-4 \alpha_{\nu} \sin ^{2} u / 2\right\}$ and $(*)$ follows by reciprocation and expansion in powers of $\sin ^{2} u / 2$. 
integrations by parts, to be

$$
\int_{-\infty}^{\infty} e^{-x s} g_{k}(x) d x=\frac{1}{s^{k}} e^{t s^{2} / 4} \quad(\mathrm{R} s>0),
$$

whence

$$
\int_{-\infty}^{\infty} e^{-x s} g_{k}(x+h) d x=\frac{1}{s^{k}} e^{t s^{2} / 4} e^{h s} \quad(\mathrm{R} s>0) .
$$

From these relations we draw two conclusions: 1 . Replacing $s$ by $s+1$ in (4.18) we obtain

$$
\int_{-\infty}^{\infty} e^{-x s} e^{-x} g_{k}(x) d x=\frac{1}{(s+1)^{k}} e^{\left(t s^{2}+2 t s+t\right) / 4} \quad(\mathrm{R} s>-1) .
$$

The right-hand side being a reciprocal $1 / \Psi(s)$, where $\Psi(s)$ is of the form (3.4), we conclude (Article 3.2) that $g_{k}(x) e^{-x}$ is a Pólya frequency function and hence:

The function $g_{k}(x)$ is totally positive.

2. Writing $h=1 / 2, h=-1 / 2$ in (4.19) and subtracting one of the equations so obtained from the other, we find

$$
\int_{-\infty}^{\infty} e^{-x s} \delta g_{k}(x) d x=\frac{1}{s^{k}} e^{t s^{2} / 4} 2 \sinh s / 2,
$$

where $\delta$ is the symbol of central differencing with unit step. On repeating the operation altogether $k$ times we find that

$$
\int_{-\infty}^{\infty} e^{-x s} \delta^{k} g_{k}(x) d x=\frac{1}{s^{k}} e^{t s^{2} / 4}(2 \sinh s / 2)^{k} .
$$

The transform so obtained agrees with (4.15) and we obtain the relation $^{12}$

$$
M_{k}(x, t)=\delta^{k} g_{k}(x) .
$$

For integral $x=n$ we obtain from (4.21)

$$
M_{k}(n, t)=\delta^{k} g_{k}(n)=\Delta^{k} g_{k}(n-k / 2),
$$

a relation which is equivalent to the identity

$$
\sum_{-\infty}^{\infty} M_{k}(n, t) z^{n}=(1-z)^{k} \sum_{n=-\infty}^{\infty} g_{k}\left(n+\frac{k}{2}\right) z^{n} \quad(0<|z|<1) .
$$

12 See [31, Part A, p. 85] for a different derivation of the relation (4.21) which is used there to construct tables of values of $M_{k}(x, t)$. 
Replacing the left-hand side by $F(z)$, in view of (4.16), and replacing $z$ by $z / 2$ we obtain

$$
\sum_{-\infty}^{\infty} \frac{1}{2^{n}} g_{k}\left(n+\frac{k}{2}\right) z^{n}=\frac{F(z / 2)}{(1-z / 2)^{k}} \quad(0<|z|<2) .
$$

From (4.20) we conclude that $\left\{g_{k}(n+k / 2)\right\}$ is a totally positive sequence. Therefore $\left\{2^{-n} g_{k}(n+k / 2)\right\}$ is a totally positive sequence which is also normalized. By Edrei's theorem the left-hand side of (4.22) can be continued into the domain $0<|z|<\infty$ as a function of the form (4.4), (4.5). On the other hand, the right-hand side of (4.22) is another such representation valid throughout the region $0<|z|$ $<\infty$, having as only singularity the pole $z=2$ of exact order $k$. On comparing these two analytic continuations of the left-hand side of (4.22) we conclude that $F(z / 2)$ is of the form (4.9) and therefore so is $F(z)$. However, the Laurent expansion (4.16) of $F(z)$ being symmetric, we conclude that $F(z)$ allows a representation of the form (4.10), hence

$$
F(z)=C e^{a\left(z+z^{-1}\right)} \prod_{1}^{\infty}\left(1+\alpha_{\nu} z\right)\left(1+\alpha_{\nu} z^{-1}\right),
$$

where $C>0, a \geqq 0,1 \geqq \alpha_{1} \geqq \alpha_{2} \cdots \geqq 0, \sum \alpha_{\nu}<\infty$. A proof of (4.17) will be complete as soon as we show that the constants satisfy the following conditions:

1. $a=0$.

2. $\alpha_{1}<1$.

3. $\alpha_{\nu}>0$ for $\nu=1,2, \cdots$.

Proof of 1 . If we denote by $M_{k}(x)$ the convolution of $k$ functions all identical with $M_{1}(x)$, defined by (4.11), then

$$
M_{k}(x, t)=\frac{1}{(\pi t)^{1 / 2}} \int_{-\infty}^{\infty} e^{-(x-u)^{2} t^{-1}} M_{k}(u) d u .
$$

However, $M_{k}(u)=0$ if $|u|>k / 2$, hence if $x>k / 2$ we find

$$
\begin{aligned}
M_{k}(x, t) & =\frac{1}{(\pi t)^{1 / 2}} \int_{-k / 2}^{k / 2} e^{-(x-u)^{2} t^{-1}} M_{k}(u) d u \\
& <\frac{1}{(\pi t)^{1 / 2}} \int_{-k / 2}^{k / 2} M_{k}(u) d u \cdot e^{-(x-k / 2)^{2} t^{-1}}
\end{aligned}
$$

or

$$
M_{k}(x, t)<\frac{1}{(\pi t)^{1 / 2}} e^{-(x-k / 2)^{2} t^{-1}} \quad \text { if } x>k / 2 .
$$


For appropriate positive constants $C_{1}$ and $\gamma\left(\gamma<t^{-1}\right)$ we therefore have $M_{k}(n, t)<C_{1} e^{-\gamma n^{2}}$, for all $n$, and (4.16) implies the estimate

$$
F(z)<2 C_{1} \sum_{0}^{\infty} e^{-\gamma n^{2}} z^{n}, \quad \text { if } z>1 .
$$

However, if $z>1$, all factors of (4.23) are $\geqq 1$ so that a fortiori

$$
e^{a_{z}}<2 C_{1} \sum_{0}^{\infty} e^{-\gamma n} z^{n}, \quad \text { if } z>1 .
$$

Since on the right side we have an entire function of order $\rho=0$, we must indeed have that $a=0$.

Proof of 2. It suffices to show that $F(-1)>0$. This last inequality can be derived as follows: Setting $s=i u$ in (4.15) we obtain

$$
\int_{-\infty}^{\infty} e^{-i x u} M_{k}(x, t) d x=e^{-t u^{2} / 4}\left(\frac{2 \sin u / 2}{u}\right)^{k} \equiv \psi_{k}(u)
$$

and by Poisson's summation formula we obtain the relation

$$
\sum_{n=-\infty}^{\infty} M_{k}(n, t) e^{i n u}=\sum_{\nu=-\infty}^{\infty} \psi_{k}(u+2 \pi v)
$$

Setting $u=\pi$, we obtain

$$
F(-1)=\left(\frac{2}{\pi}\right)^{k} \sum_{\nu=-\infty}^{\infty}(-1)^{\nu k} \frac{1}{(2 \nu+1)^{k}} e^{-t \pi(2 \nu+1) / 4} .
$$

This is visibly positive if $k$ is even. For odd values of $k$ we write it in the form

$$
\left(\frac{\pi}{2}\right)^{k} F(-1)=\left(\sum_{\nu=0}^{\infty}+\sum_{\nu=-1}^{-\infty}\right)(-1)^{\nu} \frac{1}{(2 \nu+1)^{k}} e^{-t \pi^{2}(2 \nu+1)^{2} / 4}
$$

and find that each of the two ordinary infinite series indicated has terms decreasing in absolute values and alternating sign. Thus the sums of these series are positive and again $F(-1)>0$.

PROOF OF 3. If only a finite number of $\alpha_{\nu}$ in (4.17) were positive, then $F(z)$ would be rational, hence $z=0$ would be a polar singularity for the right-hand side of (4.22). However, the Laurent expansion on the left-hand side of (4.22) shows that $z=0$ is actually an essential singularity. This contradiction completes a proof of the factorization (4.17).

A concluding remark on this subject is as follows. In [31, p. 79], it was pointed out that 


$$
f(x)=\sum_{\nu=-\infty}^{\infty} y_{\nu} M_{k}(x-\nu, t)
$$

is an analytic approximation to the given sequence of ordinates $\left\{y_{n}\right\}$ such that

$$
f(n)=\sum_{-\infty}^{\infty} y_{\nu} M_{k}(n-\nu, t)
$$

is a smoothing operation in the sense of $\$ 1$. We can now make the stronger assertion that this last operation is variation-diminishing. Indeed, as already pointed out, the sequence $a_{n}=M_{k}(n, t)$ is totally positive, a fact equivalent to our assertion (see Article 4.1).

4.4. A few unsolved problems. 1. Our first question concerns the proof of Edrei's theorem. The ties between the totally positive functions (\$3) and totally positive sequences (\$4) are apparently not as close as to allow a proof of Edrei's theorem along the lines used in characterizing Laplace transforms of Pólya frequency functions. Is this really in the nature of things or could an "elementary" proof of Edrei's theorem be devised, at least for the case when (4.6) is a power series?

2. It was pointed out in Article 4.2 that if $\Lambda(x)$ is a Pólya frequency function, then

$$
a_{n}=\Lambda(n) \quad(-\infty<n<\infty)
$$

is a totally positive normalized sequence. However, not all such sequences can be obtained in this way. Indeed, let $\left\{a_{n}\right\}$ be a totally positive sequence such that its generating Laurent series converges in $0<|z|<\infty$, and hence is of the form (4.9). However, let it be different from the series (4.7). Such a sequence can not be obtained by (4.24), by interpolating a Pólya frequency function $\Lambda(x)$. The reason for this is very simple: Assuming (4.24) to hold, $\Lambda(x)$ would have a Laplace transform (3.8) which is entire. However, (3.8) and (3.4) show that the only Pólya frequency function having an entire transform is the normal frequency function. This situation raises the question: What is the nature of totally positive sequences obtained by (4.24) from Pólya frequency functions?

3. Let us apply the integral transformation (3.10) to a bounded function $f(x)$ of period $2 \pi$. Setting

$$
\chi(x)=\sum_{n=-\infty}^{\infty} \Lambda(x+2 \pi n)
$$


the transformation (3.10) becomes

$$
g(x)=\int_{0}^{2 \pi} \chi(x-t) f(t) d t
$$

which shows that $g(x)$ is continuous and of period $2 \pi$. Assuming $\Lambda(x)$ to be a Pólya frequency function, (4.26) implies that $v(g) \leqq v(f)$ (see Article 3.3); however, this relation is now meaningless since $v(g)$ and $v(f)$ either vanish or else are infinite. It is not difficult to see that (4.26) now enjoys the variation-diminishing property in the cyclic sense, i.e. it implies

$$
v_{c}(g) \leqq v_{c}(f),
$$

where these numbers of changes of sign are counted on the circle, or within a period. The special case of $(4.26)$ when

$$
\Lambda_{\tau}(x)=\frac{1}{(\pi \tau)^{1 / 2}} e^{-x^{2} / \tau} \quad(\tau>0),
$$

was already noticed by Pólya [20], not in its integral form (4.26) but rather in terms of the equivalent convolution of the respective Fourier series $;^{13} \chi(x)$ then becomes the Fourier kernel for a ring known from heat-conduction.

The following question arises: What is the nature of the periodic functions $\chi(x)$ with the property that the transformation (4.26) is cyclically variation-diminishing, i.e. implies (4.27)? Our formula (4.25) furnishes such functions but does not produce all of them. Indeed

$$
\chi(x)=a+\cos t
$$

( $a$ constant $\geqq 1)$

is readily shown to have the property (4.27), without, however, being of the form (4.25).

Concluding, let us briefly mention a connection between the transformations (4.26) and the convex curves of Article 2.2. Let us assume

$$
\int_{0}^{2 \pi} \chi(t) d t=1
$$

and let $C$, defined by (2.8), be convex in $E_{2 n}$. Then the new curve

$$
\begin{aligned}
\bar{C}: \quad x_{i}=\bar{x}_{i}(x)=\int_{0}^{2 \pi} & \chi(x-t) x_{i}(t) d t \\
& (i=1, \cdots, 2 n ; 0 \leqq x \leqq 2 \pi)
\end{aligned}
$$

${ }^{13}$ For yet another example of a special function $\chi(x)$ see Pólya-Wiener [27, Lemma 1]. 
is also convex in $E_{2 n}$. Indeed, (4.29) and (4.30) imply the relation

$$
A_{0}+\sum_{1}^{2 n} A_{i} \bar{x}_{i}(x)=\int_{0}^{2 \pi} \chi(x-t)\left(A_{0}+\sum_{1}^{2 n} A_{i} x_{i}(t)\right) d t
$$

and by (4.27):

$$
v_{c}\left(A_{0}+\sum A_{i} \bar{x}_{i}(t)\right) \leqq v_{c}\left(A_{0}+\sum A_{i} x_{i}(t)\right) .
$$

This relation means that $\bar{C}$ crosses a hyperplane not more often than $C$ does, hence at most $2 n$ times.

If we choose in particular the Fourier kernel $\chi_{\tau}(x)$ arising from (4.25) and $\Lambda(x)=\Lambda_{\tau}(x)$, defined by (4.28), then we see that the $2 n$ functions (4.30) are analytic (even entire) functions of the parameter $x$. As $\tau \rightarrow+0$, we evidently have $\bar{x}_{i}(x) \rightarrow x_{i}(x)$. This remark proves the following: A curve convex in $E_{2 n}$ is the limit of a family of curves convex in $E_{2 n}$ which are analytic.

Added in proof, April 1953. Professor W. Fenchel kindly pointed out to me that also Gantmakher and Krein have been essentially anticipated in the discovery of their theorem of Article 2.2 by the late J. Hjelmslev in his paper Introduction à la théorie des suites monotones, Oversigt over det Kgl. Danske Videnskabernes Selskabs Forhandlinger, 1914, pp. 1-74. Indeed Hjelmslev gives analytic conditions in order that the arc $\Gamma$ in $E_{m}$, defined by (2.5), be of order $m$. The precise statement of his result (loc. cit. p. 62) is as follows: If no subarc of $\Gamma$ is in $E_{m-1}$, then $\Gamma$ is of order $m$ if and only if all determinants (2.6) are positive, or all these determinants are negative. Arcs satisfying these determinant conditions are called monotone arcs by Hjelmslev.

\section{BIBLIOGRAPHY}

1. E. Laguerre, Sur les fonctions de genre zéro et du genre un, C. R. Acad. Sci. Paris vol. 95 (1882) pp. 828-831 [also Oeuvres, vol. I, pp. 174-177].

2. J. Hadamard, Essai sur l'étude des fonctions données par leur développement de Taylor, Journal de Mathématiques (4) vol. 8 (1892) pp. 101-186.

3. A. Hurwitz, Sur le problème des isopérimetres, C. R. Acad. Sci. Paris vol. 132 (1901) pp. 401-403 [also Werke, vol. I, pp. 490-491].

4. M. Fekete, Über ein Problem von Laguerre, Rendiconti di Palermo vol. 34 (1912) pp. 1-12.

5. G. Pólya, Über Annäherung durch Polynome mit lauter reellen Wurzeln, Rendiconti di Palermo vol. 36 (1913) pp. 1-17.

6. J. Grommer, Ganze transzendente Funktionen mit lauter reellen Nullstellen, Journal für Mathematik vol. 144 (1914) pp. 114-165.

7. G. Pólya and I. Schur, Über zwei Arten von Faktorenfolgen in der Theorie der algebraischen Gleichungen, Journal für Mathematik vol. 144 (1914) pp. 89-113.

8. G. Pólya, Algebraische Untersuchungen über ganze Funktionen vom geschlechte Null und Eins, Journal für Mathematik vol. 145 (1915) pp. 224-249. 
9. O. D. Kellogg, The oscillation of functions of an orthogonal set, Amer. J. Math. vol. 38 (1916) pp. 1-5.

10. - Orthogonal function sets arising from integral equations, Amer. J. Math. vol. 40 (1918) pp. 144-154.

11. - Interpolation properties of orthogonal sets of solutions of differential equations, Amer. J. Math. vol. 40 (1918) pp. 225-234.

12. H. Hamburger, Bemerkungen zu einer Fragestellung des Herrn Pólya, Math. Zeit. vol. 7 (1920) pp. 302-322.

13. G. P6́lya, On the mean-value theorem corresponding to a given linear homogeneous differential equation, Trans. Amer. Math. Soc. vol. 24 (1922) pp. 312-324.

14. G. Pólya and G. Szegö, Aufgaben und Lehrsätze aus der Analysis, vol. II, Berlin, 1925.

15. H. H. Wolfenden, On the development of formulae for graduation by linear compounding, with special reference to the work of Erastus L. De Forest, Transactions of the Acturial Society of America vol. 26 (1925) pp. 81-121.

16. S. Bernstein, Lę̧ons sur les propriêtés extrémales des fonctions analytiques, Paris, 1926.

17. E. T. Whittaker and G. Robinson, The calculus of observations, 2d ed., London and Glasgow, 1926.

18. A. Marchaud, Sur les continus d'ordre borné, Acta Mathematica vol. 55 (1930) pp. 67-115.

19. I. J.Schoenberg, Über variationsvermindernde lineare Transformationen, Math. Zeit. vol. 32 (1930) pp. 321-328.

20. G. Pólya, Qualitatives über Wärmeausgleich, Zeitschrift für Angewandte Mathematik und Mechanik vol. 13 (1933) pp. 125-128.

21. I. J. Schoenberg, Zur Abzählung der reellen Wurzeln algebraischer Gleichungen, Math. Zeit. vol. 38 (1934) pp. 546-564.

22. T. S. Motzkin, Beiträge zur Theorie der linearen Ungleichungen, dissertation, Basel, 1933, Jerusalem, 1936.

23. A. Wintner, On a class of Fourier transforms, Amer. J. Math. vol. 58 (1936) pp. $45-90$.

24. F. Gantmakher and M. Krein, Sur les matrices complètement non négatives et oscillatoires, Compositio Math. vol. 4 (1937) pp. 445-476.

25. P. Scherk, Über differenzierbare Kurven und Bögen, Casopis pro pěstovani Matematiky a Fysiky vol. 66 (1937) pp. 165-191.

26. G. B. Dantzig, On a class of distributions that approach the normal distribution function, Ann. Math. Statist. vol. 10 (1939) pp. 247-253.

27. G. Pólya and N. Wiener, On the oscillation of the derivatives of a periodic function, Trans. Amer. Math. Soc. vol. 52 (1942) pp. 249-256.

28. O. Szász, On sequences of polynomials and the distribution of their zeros, Bull. Amer. Math. Soc. vol. 49 (1943) pp. 377-383.

29. T. N. E. Greville, The general theory of osculatory interpolation, Transactions of the Actuarial Society of America vol. 45 (1944) pp. 202-265.

30. H. Rademacher and I. J. Schoenberg, An iteration method for calculation with Laurent series, Quarterly of Applied Mathematics vol. 4 (1946) pp. 142-159.

31. I. J. Schoenberg, Contributions to the problem of approximation of equidistant data by analytic functions, Quarterly of Applied Mathematics vol. 4 (1946) Part A pp. 45-99, Part B, pp. 112-141.

32. D. V. Widder, The Laplace transform, Princeton, 1946.

33. H. B. Curry and I. J. Schoenberg, On Pólya frequency functions IV: The spline 
functions and their limits, as yet unpublished; see Bull. Amer. Math. Soc. Abstract 53-11-380.

34. I. J. Schoenberg, On totally positive functions, Laplace integrals and entire functions of the Laguerre-Pólya-Schur type, Proc. Nat. Acad. Sci. U.S.A. vol. 33 (1947) pp. 11-17.

35. D. V. Widder, Inversion formulas for convolution transforms, Duke Math. J. vol. 14 (1947) pp. 217-249.

36. W. Feller, On the Kolmogorov-Smirnov limit theorems for empirical distributions, Ann. Math. Statist. vol. 19 (1948) pp. 177-189.

37. I. I. Hirschman and D. V. Widder, The inversion of convolution transform with totally positive kernels, Proc. Nat. Acad. Sci. U.S.A. vol. 34 (1948) pp. 152-156.

38. I. J. Schoenberg, Some analytical aspects of the problem of smoothing, Courant Anniversary volume "Studies and Essays," New York, 1948, pp. 351-370.

39. - On variation-diminishing integral operators of the convolution type, Proc. Nat. Acad. Sci. U.S.A. vol. 34 (1948) pp. 164-169.

40. I. I. Hirschman and D. V. Widder, The inversion of a general class of convolution transforms, Trans. Amer. Math. Soc. vol. 66 (1949) pp. 135-201.

41. - A representation theory for a general class of convolution transforms, Trans. Amer. Math. Soc. vol. 67 (1949) pp. 69-97.

42. P. Laasonen, Einige Sätze über Tschebycheffsche Systeme, Ann. Acad. Sci. Fennicae, Ser. A. I. Math. Phys. no. 52, 24 pp., 1949.

43. I. J. Schoenberg and Anne Whitney, Sur la positivité des determinants de translations des fonctions de fréquence de Pólya . . . , C. R. Acad. Sci. Paris vol. 228 (1949) pp. 1996-1998.

44. F. Gantmakher and M. Krein, Oscillatory matrices and kernels and small vibrations of mechanical systems (in Russian), 2d ed., Moscow, 1950.

45. I. I. Hirschman, Proof of a conjecture of I. J. Schoenberg, Proc. Amer. Math. Soc. vol. 1 (1950) pp. 63-65.

46. I. J. Schoenberg, On Pólya frequency functions II: Variation-diminishing integral operators of the convolution type, Acta Sci. Math. Szeged vol. 12 (1950) pp. 97106.

47. - The finite Fourier series and elementary geometry, Amer. Math. Monthly vol. 57 (1950) pp. 390-404.

48. M. Aissen, A. Edrei, I. J. Schoenberg, and Anne Whitney, On the generating functions of totally positive sequences, Proc. Nat. Acad. Sci. U.S.A. vol. 37 (1951) pp. 303-307.

49. F. John, On integration of parabolic equations by difference methods, Communications on Pure and Applied Mathematics vol. 5 (1952) pp. 155-211.

50. T. S. Motzkin and I. J. Schoenberg, On lineal entire functions of $n$ complex variables, Proc. Amer. Math. Soc. vol. 3 (1952) pp. 517-526.

51. I. J. Schoenberg and Anne Whitney, $A$ theorem on polygons in $n$ dimensions with applications to variation-diminishing and cyclic variation-diminishing linear transformations, Compositio Math. vol. 9 (1951) pp. 141-160.

52. D. V. Widder, Weierstrass transforms of positive functions, Proc. Nat. Acad. Sci. U.S.A. vol. 37 (1951) pp. 315-317.

53. - Necessary and sufficient conditions for the representation of a function by a Weierstrass transform, Trans. Amer. Math. Soc. vol. 71 (1951) pp. 430-439.

54. J. Berghuis, $A$ class of entire functions used in analytic interpolation, Proceedings Royal Neth. Acad. of Sciences. Ser. A vol. 55 (1952) pp. 468-473.

55. I. J. Schoenberg, On Pólya frequency functions I: The totally positive functions 
and their Laplace transforms, Journal d'Analyse Mathématique vol. 1 (1951) pp. 331374; actually appeared in 1952.

56. —- An isoperimetric inequality for closed curves convex in even-dimensional euclidean spaces, to appear in the Acta Math.

57. I. J. Schoenberg and Anne Whitney, On Pólya frequency functions III: The positivity of translation determinants with an application to the interpolation problem by spline curves, Trans. Amer. Math. Soc. vol. 74 (1953) pp. 246-259.

58. Anne Whitney, A reduction theorem for totally positive matrices, Journal d'Analyse Mathématique vol. 2 (1952) pp. 88-92.

59. M. Aissen, I. J. Schoenberg, and A. Whitney, On the generating functions of totally positive sequences I, Journal d'Analyse Mathématique vol. 2 (1952) pp. 93-103.

60. A. Edrei, On the generating functions of totally positive sequences II, Journal d'Analyse Mathématique vol. 2 (1952) pp. 104-109.

61. - Proof of a conjecture of Schoenberg on the generating function of a totally positive sequence, Canadian Journal of Mathematics vol. 5 (1953) pp. 86-94.

62. - On the generating function of doubly infinite, totally positive sequences, submitted for publication in Trans. Amer. Math. Soc.

University of California, Los Angeles, and University of Pennsylvania 